\title{
Evaluating associations between Mediterranean diet adherence indexes and biomarkers of diet and disease
}

\author{
A Bach-Faig 1,3* , D Geleva ${ }^{2}$, JL Carrasco ${ }^{3}$, L Ribas-Barba ${ }^{1}$ and L Serra-Majem ${ }^{1}$ \\ ${ }^{1}$ Mediterranean Diet Foundation, University of Barcelona Science Park, Barcelona, Spain: ${ }^{2}$ Department of Family \\ and Consumer Sciences, Seattle Pacific University, Seattle, WA, USA: ${ }^{3}$ Biostatistics, Department of Public Health, \\ University of Barcelona, Barcelona, Spain
}

Submitted 3 April 2006: Accepted 6 November 2006

\begin{abstract}
Objective: We examined associations between two Mediterranean diet (MD) adherence indexes (the MD index, MDI, and the MD score, MDS) and several blood biomarkers of diet and disease.

Subjects: We studied 328 individuals from Catalonia (Northeastern Spain), ages 1875 , who provided fasting blood samples, a subset of the 2346 individuals as part of a larger representative and random sample from the 1992-1993 Catalan Nutritional Survey.

Design and method: Diet was measured using 24-h recalls. Biomarkers studied were plasma levels of $\beta$-carotene, $\alpha$-tocopherol, retinol, vitamins B12, C and folates as well as serum total cholesterol, HDL cholesterol, LDL cholesterol and triglycerides. Multivariate linear regression was used to analyse associations of the nutrient biomarkers with the dietary pattern indexes, adjusting for potential confounders. Results and conclusions: Subjects with higher MD adherence, as measured by the two dietary indexes, had significantly higher plasma concentrations of $\beta$-carotene, folates, vitamin C, $\alpha$-tocopherol and HDL cholesterol. The most highly significant relationship was that between folates and the adherence to the MD Pattern, as determined by both indexes. These research findings suggest the potential usefulness of biomarkers as complementary tools for assessing adherence to a dietary pattern. This type of data not only informs the development of robust dietary adherence indexes, but it also provides specific clues about the potential physiological mechanisms that explain the beneficial effects of the MD pattern on chronic disease risk.
\end{abstract}

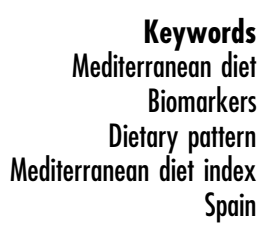

\section{Introduction}

Numerous studies have supported the beneficial effect of the Mediterranean diet pattern (MDP) on chronic disease risk (cardiovascular disease, diabetes mellitus and several types of cancer $^{1}$ ), since the pattern was identified in the $1960 \mathrm{~s}^{2}$.

Epidemiological studies, evaluating the relationship between specific nutrients or single foods and various chronic disease risk factors are common. Such a reductionist approach, however, is problematic because nutrients and foods are not consumed in isolation and thus, their effect is likely cumulative, if not synergistic ${ }^{3,4}$. This belief has led to an increasing number of studies over the past decade which attempt to evaluate the effect that dietary patterns have on health ${ }^{3,5}$.

Adopting the MDP can be a holistic and realistic strategy to changing an individual's diet as well as beneficially impacting public health. Various scores or indexes have been used to assess the adherence to an MDP in several populations around the world ${ }^{6}$. Such dietary indexes attempt to summarise the complexity of a pattern generally characterised by a high and varied plant food intake (e.g. vegetables, fruits, legumes, nuts, seeds and cereal grains (especially wholemeal) a relatively low meat and animal food intake, use of olive oil as a main source of fat, a moderate intake of fish and poultry, a moderate to low intake of dairy products (mainly as yogurt or cheese), as well as moderate consumption of wine with meals ${ }^{7}$. Using such an index, individual diet quality can be ranked and this has proven a useful tool in the study of diet and health relationships ${ }^{8}$.

Biomarkers have commonly been used to assess nutritional status and dietary intake, to evaluate health and predict disease risk ${ }^{9,10}$. Even though biomarker data have long been used in the above-described ways, only recently it has been suggested as a useful tool in describing dietary patterns. This is because of the ability of such data not only to provide a more objective and complementary 
measure of dietary intake than data obtained with traditional nutritional assessment tools, but also to offer insight into mechanisms by which the MDP could influence disease development and processes ${ }^{10,11}$.

The aim of the present study was to examine the associations between two Mediterranean diet adherence indexes and several blood biomarkers. Specifically, we examined plasma levels of several vitamins ( $\beta$-carotene, $\alpha$-tocopherol, retinol, vitamins B12 and C, and folates) and serum lipid concentrations (total cholesterol, HDL cholesterol, LDL cholesterol and triglycerides). We hypothesised that higher consumption of plant foods over animal foods, olive oil use as well as the rest of the elements of the MDP, may be associated with higher levels of these vitamin biomarkers as well as a more healthful lipid profile.

\section{Materials and methods}

\section{Study sample}

This study analysed data from a sample of 328 men and women, ages 18-75. This was a subset of the 2346 individuals who provided blood samples as part of a larger representative and random sample of the Catalan population (Northeast Spain), participating in a 19921993 Nutritional Survey ${ }^{12}$.

This survey of the nutritional status of the Catalan population was a cross-sectional descriptive study that was conducted as part of the Food and Nutrition Programme of the Public Health Office (Department of Health) of the Catalan Government and was coordinated by the Community Nutrition Research Centre of the University of Barcelona ${ }^{12}$.

\section{Measures}

\section{Blood collection and assessment of biomarkers}

The biomarkers examined were plasma levels of several vitamins, including $\beta$-carotene, $\alpha$-tocopherol, retinol, vitamins B12 and $\mathrm{C}$, and several folates as well as serum lipid concentrations, including total cholesterol, HDL cholesterol, LDL cholesterol and triglycerides.

Blood samples were collected between January and July 1993. All biochemistry procedures utilised have been explained in detail previously ${ }^{13,14,15}$. The biochemical parameters were determined from vacuum-extracted blood samples, collected at the same time of day (from 8 to 10 in the morning) after a 12 -h fast. The blood samples were centrifuged at $3000 \mathrm{rpm}$ for $15 \mathrm{~min}$ at $8^{\circ} \mathrm{C}$, after a previous 30 -min centrifugation, aliquoted and protected from light and heat throughout processing and storage.

Lipid aliquot samples were kept at $4^{\circ} \mathrm{C}$ and were analysed within $24 \mathrm{~h}$ of extraction. The aliquots for the rest of the biomarkers ( $\beta$-carotene, $\alpha$-tocopherol, retinol, vitamin $\mathrm{B} 12$ and folates) were stored at $-80^{\circ} \mathrm{C}$ before processing. Serum ascorbic acid (vitamin C) samples, before being frozen, were preserved by the addition of $2 \mathrm{ml}$ of metaphosphoric acid/dithiothretol solution (6\%) for each millilitre of serum.

Standard laboratory procedures were carried for the measurement of the selected biomarkers. Vitamin B12, and folic acid were analysed by a radioimmunoassay (RIA), using the B12/Folate ComoStat II kit. Serum vitamin C specimens were determined by the 2,4-dinitrophenylhydrazine colorimetric procedure. Lipid concentrations (total cholesterol and triglycerides) were analysed with enzymatic procedures. The HDL cholesterol concentrations were analysed by precipitation and the LDL cholesterol was calculated using the Friedewald equation ${ }^{13}$. Serum vitamins, such as $\beta$-carotene, $\alpha$-tocopherol and retinol, were measured by high-performance liquid chromatography (HPLC). Both $\alpha$-tocopherol and retinol concentrations were tightly correlated with blood lipid concentrations (cholesterol and triglycerides) and were, therefore, standardised for them $^{12,16}$.

\section{Diet assessment}

Dietary interviewers who had undergone special selection and training collected dietary data from the complete sample by means of home interviews, using two 24-h recalls. In order to avoid seasonal influences, first recall was done between May and July, and a second one during the months of November and December ${ }^{12}$.

In order to facilitate the interpretation and description of portion sizes, in most cases, common household measurements were used. The information collected was quantified, coded and analysed for the amounts of food. For this study, energy and nutrient content information was obtained using Spanish food composition tables ${ }^{17}$.

\section{Mediterranean diet pattern adberence indexes}

Two MDIs were applied to the 24-h recall data: a variant of the Sánchez-Villegas et al. index ${ }^{18}$ that adds or subtracts standardised food components and the Trichopoulou et al. Mediterranean diet score based on either positive or negative scoring of the components ${ }^{19}$.

In the first MDI computed, the adherence to the MDP was defined through 10 characteristics, using the calculation methodology of Sánchez-Villegas et al. ${ }^{18}$ and Tur et $a l^{20}$, but with small component modifications. Each individual adherence value was expressed as a percentage and calculated by adding and subtracting (depending on their positive or negative effect on health) the standardised food components, as follows:

$$
\begin{aligned}
\Sigma Z_{i}= & Z_{\text {legumes }}+Z_{\text {cereals }}+Z_{\text {fruits }}+Z_{\text {vegetables }}+Z_{\text {fish }} \\
& +Z_{\text {moderate alcohol }}+Z_{\text {nuts }}+Z_{\text {MUFA:SFA }} \\
& -Z_{\text {meat and derivatives }}-Z_{\text {milk and derivatives }}
\end{aligned}
$$

Legumes, cereals, fruits, vegetables (including tubers), fish, moderate alcohol, nuts and monounsaturated/ saturated fatty acid (MUFA/SFA) ratio were considered 
the protective components, while meat and meat derivatives as well as milk and dairy products (mainly high in fat) the non-protective components. As discussed by Tur $e t a l{ }^{20}$, a standardised healthful consumption limit was used to define 'moderate' alcohol consumption. Alcohol intake lower than 20 and $30 \mathrm{~g} \mathrm{day}^{-1}$ for women and men, respectively, was given the highest value because most of the studies demonstrate the greatest coronary disease risk reduction at these levels of intake ${ }^{21,22}$. Progressively lower values were assigned when consumption was much lower than or exceeded these values ${ }^{20}$.

The MDI was transformed to relative percentage of adherence using the range of values in the sample, thus resulting in maximum adherence of 100 and a minimum adherence of zero:

$$
\text { Adherence }(\%)=\frac{\left(\Sigma Z_{i}-\Sigma Z_{\min }\right) \times 100}{\left(\Sigma Z_{\max }-\Sigma Z_{\min }\right)}
$$

The other index applied was the Mediterranean diet score (MDS), in which the MD is simplified to 10 components, respectively ${ }^{19}$.

The MDS was generated by assigning a score from 0 to 1 based on the daily intake of the various components. The sample medians of the daily consumption of these components, in grams and by sex, were used as cut-off points.

In the MDS, subjects received a point if their intake was higher than the sample median for a protective component (legume, grain, fruit and vegetable intake, MUFA/SFA ratio, fish and moderate poultry intake) and lower than the median for a non-protective component (meat and meat products, as well as milk and dairy products). An exception was alcohol, in which case 1 point was given to males if their consumption was within a $10-50 \mathrm{~g} \mathrm{day}^{-1}$ range and to women if consumption was within 5-25 g day $^{-1}$.

If an individual incorporated all the components of the diet, they would obtain the highest possible score and this would reflect a greater adherence to the MD. The MDS ranges from 0 (minimal adherence) to 10 (maximum adherence) because the index has 10 components.

\section{Assessment of other variables}

Socioeconomic, demographic, anthropometric and lifestyle (physical activity, smoking habits, etc.) data were also collected using a sociodemographic questionnaire.

Tobacco consumption was assessed following the World Health Organisation classification norms ${ }^{23}$.

Anthropometric measurements, such as weight, height and waist circumference, were taken using standard measurement equipment. From these, the body mass index (BMI) was calculated for each subject.

In order to evaluate physical activity, subjects were asked if they participated in physical activities or sports in their leisure time, but only free-time activities outside the workplace were considered.

\section{Statistical analysis}

Statistical analyses were performed using SPSS-PC, Version 12.0 (SPSS Inc., Chicago, IL, USA) and conducted through regression models. Multiple linear regression models were applied to assess associations between the MD adherence indexes and biomarkers, including plasma levels of several vitamins and serum lipid concentrations. To examine the trends in biomarker concentrations, the dietary pattern indexes were modelled as a continuous dependent variable. An association was considered significant if the $P$-value was lower than $5 \%$.

Covariates potentially related to MDP and the examined biomarkers were chosen on an a priori basis and included gender, age, BMI and lifestyle factors, such as current smoking status and frequency, free-time physical activity, total energy intake and presence of chronic disease (diabetes mellitus type I or II and hypertension). Adjustments for the covariates with significant $P$-values $(<0.1)$, including sex, age and energy intake in the MDI analysis and age and energy in the MDS were made.

Measures of central tendency (averages) and dispersion (standard deviations) for each biomarker were obtained according to the groups created by approximate quartiles for the MDP adherence index.

\section{Results}

The mean age of the 328 participants in this study was 44.8 years, with a standard deviation of 16.8 and there were slightly more women (58\%) than men (42\%). Smoking prevalence was around 30\%. Similar to the larger sample of which this one was a subset, half of the individuals resided in big cities ( $>100000$ inhabitants) and the rest in middle-size (10 000-100 000 inhabitants) and small-size $(<10000)$ towns. Sixty-four per cent of the participants were sedentary based on leisure-time activity (Table 1).

The mean MDP adherence based on MDI and MDS was $48 \pm 14 \%$ and $4.77 \pm 1.7 \%$, respectively, (mean \pm standard deviation).

Means and standard deviations of biomarkers by quartiles of adherence to the MDP, and adjusted Pearson's correlations and $P$-values are presented in Table 2. Subjects with higher MDP adherence, as measured by the two indexes, had significantly higher plasma concentrations of vitamin $\mathrm{C}$ and folates. When using the MDI and MDS indexes, $\beta$-carotene was also significantly related to the MDP adherence. Additionally, when applying the MDS, HDL cholesterol levels were significantly associated with MDP adherence. Lastly, subjects in the fourth quartile of MDP adherence had significantly higher serum standardised $\alpha$-tocopherol concentrations 
Table 1 Demographic and lifestyle characteristics of the sample $(n=328)$

\begin{tabular}{|c|c|c|}
\hline Characteristic & $n$ & $\%$ \\
\hline \multicolumn{3}{|l|}{ Age (years) } \\
\hline $18-24$ & 53 & 16 \\
\hline $25-34$ & 57 & 18 \\
\hline $35-44$ & 63 & 19 \\
\hline $45-54$ & 42 & 13 \\
\hline $55-64$ & 70 & 21 \\
\hline$>64$ & 43 & 13 \\
\hline \multicolumn{3}{|l|}{ Sex } \\
\hline Men & 137 & 42 \\
\hline Women & 191 & 58 \\
\hline \multicolumn{3}{|l|}{ Body Mass Index } \\
\hline$\leq 20$ & 27 & 8 \\
\hline $20.1-24.9$ & 141 & 43 \\
\hline $25.0-29.9$ & 121 & 37 \\
\hline$\geq 30$ & 39 & 12 \\
\hline \multicolumn{3}{|c|}{ Physical activity in leisure time } \\
\hline Yes & 109 & 36 \\
\hline No & 194 & 64 \\
\hline \multicolumn{3}{|l|}{ Tobacco use } \\
\hline No & 233 & 71 \\
\hline Yes & 95 & 29 \\
\hline \multicolumn{3}{|l|}{ Population size } \\
\hline$<10000$ & 84 & 26 \\
\hline $10000-100000$ & 73 & 22 \\
\hline$>100000$ & 171 & 52 \\
\hline
\end{tabular}

than those individuals with lower adherence (quartiles 1, 2 and 3 ) according to the MDS.

Before adjusting for confounding variables, associations between the MDP adherence indexes and total cholesterol, LDL cholesterol, triglycerides, $\alpha$-tocopherol and retinol were shown to be significant (data not shown). However, this significance disappeared once variables were adjusted.

In a complementary regression analysis, associations between individual food groups and biomarkers were also examined. As expected, protective components, such as fruits and vegetables showed a positive association with a few vitamin biomarkers ( $\beta$-carotene, vitamin $\mathrm{C}$ and folic acid) (data not shown). However, associations between food groups and biomarkers were less significant than the associations between the two dietary pattern indexes and biomarkers.

\section{Discussion}

In the present study, two adherence indexes (MDS and MDI) were used to evaluate the diet of a random sample of the Catalan population of Northeastern Spain. Results demonstrated that MDP adherence in this group was moderately good, as indicated by a mean score of four points, using the MDS. Such a value has been associated with a satisfactory MDP adherence and positive health implications $^{19}$. Subjects with higher MDP adherence, as measured by the two different dietary pattern indexes, had higher plasma concentrations of some of the examined biomarkers with presumed beneficial properties, including $\beta$-carotene, folates, vitamin C, $\alpha$-tocopherol and HDL cholesterol. The most highly significant relationship was that with folates.

A few other epidemiological studies have recently examined the relationship between dietary patterns and similar biological markers of intake and disease. Most of these have compared a general healthy or prudent dietary pattern with a Western diet pattern, by using either indexbased patterns, or patterns derived from exploratory factor analyses ${ }^{24-32}$. Index-based patterns may have an advantage because, unlike patterns derived from exploratory factor analyses, they reflect known diet and disease associations, and therefore, usually demonstrate stronger associations with biomarkers ${ }^{24}$.

Consistent with others' findings, we observed a positive correlation between some serum biomarkers of fruit and vegetable intake and the MD pattern indexes ${ }^{24,25,30}$. For instance, Newby et al. reported that plasma carotenoids and vitamin E were positively correlated with a revised diet quality index (DQI) ${ }^{31}$, while Hann et al. reported significant associations of better Healthy Eating Index Scores with higher serum concentrations of folates, ascorbic acid and all carotenoids except lycopene ${ }^{30}$. In another study, a prudent pattern was a positive predictor of serum folate; however, 15 other examined biomarkers of disease were unrelated with this pattern ${ }^{29}$. Gerber et al. found a significant correlation between DQI adapted for the French Mediterranean diet and $\alpha$-tocopherol and $\beta$-carotene ${ }^{32}$. Neuhouser et al. also found that vitamin C and $\alpha$-tocopherol were significantly associated with higher DQI scores ${ }^{26}$.

In the present study, no associations were found between the dietary pattern indexes and the blood lipid levels examined, with the exception of HDL cholesterol. In several studies, blood lipid levels were also not correlated with a healthy dietary pattern ${ }^{29}$, such as the Mediterranean $^{25,32}$. However, in other studies, serum cholesterol was found to be negatively correlated with a healthy pattern index ${ }^{31}$.

In our study, most of the examined relationships between biomarkers and the adherence indexes were stronger than those for individual food groups. Likewise, in Gerber et al., correlation coefficients for $\alpha$-tocopherol and $\beta$-carotene were as high as, or higher with the DQI than with most relevant foods ${ }^{25}$. This finding suggests that if certain nutrients, such as $\beta$-carotene or $\alpha$-tocopherol are related to decreased risk of a disease, the mentioned nutrients may not be responsible for the decreased risk, but rather, the decrease in risk could be due to a wholefood pattern of which the nutrient is only a marker.

Most researchers are well aware of the need to control for confounders $^{33}$. Age, sex, population size, BMI, smoking, physical activity, total energy intake and the presence of chronic disease are variables that might confound dietary pattern effects as shown in many previous studies ${ }^{18,20,24,25,32,34,35}$. Like diet, biomarkers may 
Table 2 Relationships between adjusted dietary indexes and the evaluated biomarkers by quartiles

\begin{tabular}{|c|c|c|c|c|c|c|}
\hline & & uartiles of adjusted & tary Pattern Indexe & & & \\
\hline & Q1 $(n=82)$ & $\mathrm{Q} 2(n=82)$ & Q3 $(n=82)$ & Q4 $(n=82)$ & & \\
\hline MDI & $<38.77$ & $38.77-48.00$ & $48.00-56.24$ & $>56.24$ & & \\
\hline MDS & $<3.63$ & $3.63-4.71$ & $4.71-5.83$ & $>5.83$ & & \\
\hline & Mean \pm sta & deviation ${ }^{1}$ of bion & ers by quartiles of & sted Dietary & correlation & $P$-values ${ }^{2}$ \\
\hline Biomarkers & & Patter & dexes & & (adjusted) & (adjusted) \\
\hline$\beta$-carotene & $\left(\Gamma^{1}\right)$ & & & & & \\
\hline MDI & $0.40 \pm 0.34$ & $0.42 \pm 0.34$ & $0.54 \pm 0.34$ & $0.45 \pm 0.29$ & 0.141 & 0.044 \\
\hline MDS & $0.37 \pm 0.29$ & $0.49 \pm 0.38$ & $0.41 \pm 0.27$ & $0.53 \pm 0.35$ & 0.153 & 0.006 \\
\hline$\alpha$-tocophero & $\left.10 / \Gamma^{-1}\right)$ & & & & & \\
\hline MDI & $33.41 \pm 10.25$ & $31.09 \pm 8.06$ & $35.44 \pm 10.36$ & $33.68 \pm 10.23$ & 0.090 & 0.225 \\
\hline MDS & $32.94 \pm 10.07$ & $31.80 \pm 8.47$ & $33.79 \pm 8.49$ & $35.05 \pm 11.73$ & 0.055 & 0.327 \\
\hline Standardise & ocopherol ( $\mu \mathrm{mol} \Gamma$ & & & & & \\
\hline MDI & $32.08 \pm 6.55$ & $30.54 \pm 5.26$ & $33.92 \pm 11.74$ & $31.49 \pm 7.07$ & 0.035 & 0.644 \\
\hline MDS & $31.79 \pm 5.76$ & $31.05 \pm 6.94$ & $30.65 \pm 4.82$ & $34.31 \pm 12.27$ & 0.115 & 0.039 \\
\hline Retinol $(\mu \mathrm{m}$ & & & & & & \\
\hline MDI & $1.84 \pm 0.53$ & $1.79 \pm 0.53$ & $1.88 \pm 0.43$ & $1.95 \pm 0.47$ & 0.025 & 0.124 \\
\hline MDS & $1.81 \pm 0.55$ & $1.87 \pm 0.51$ & $1.88 \pm .48$ & $1.89 \pm 0.42$ & 0.073 & 0.188 \\
\hline Standardise & $\mathrm{inol}\left(\mu \mathrm{mol} \Gamma^{-1}\right)$ & & & & & \\
\hline MDI & $1.82 \pm 0.56$ & $1.79 \pm 0.54$ & $1.82 \pm 0.51$ & $1.86 \pm 0.45$ & -0.038 & 0.660 \\
\hline MDS & $1.74 \pm 0.54$ & $1.85 \pm 0.53$ & $1.80 \pm 0.50$ & $1.88 \pm 0.47$ & 0.108 & 0.052 \\
\hline Vitamin $C(r$ & & & & & & \\
\hline MDI & $9.98 \pm 4.16$ & $11.80 \pm 3.71$ & $11.71 \pm 3.52$ & $10.86 \pm 3.75$ & 0.095 & 0.047 \\
\hline MDS & $10.34 \pm 4.25$ & $11.24 \pm 3.87$ & $11.01 \pm 3.84$ & $11.72 \pm 3.33$ & 0.152 & 0.006 \\
\hline Vitamin B12 & $\left.m I^{-1}\right)$ & & & & & \\
\hline MDI & $610.79 \pm 383.63$ & $556.48 \pm 216.43$ & $773.30 \pm 869.33$ & $622.91 \pm 481.83$ & 0.050 & 0.722 \\
\hline MDS & $661.62 \pm 603.98$ & $608.16 \pm 221.90$ & $710.93 \pm 844.48$ & $572.61 \pm 242.63$ & -0.025 & 0.648 \\
\hline Folate ( $\mathrm{ngm}$ & & & & & & \\
\hline MDI & $7.95 \pm 3.59$ & $8.59 \pm 3.72$ & $9.10 \pm 3.33$ & $9.58 \pm 4.53$ & 0.180 & 0.001 \\
\hline MDS & $7.84 \pm 3.76$ & $9.13 \pm 4.19$ & $8.30 \pm 3.23$ & $9.87 \pm 3.94$ & 0.164 & 0.003 \\
\hline Total choles & $\left(m g d l^{-1}\right)$ & & & & & \\
\hline MDI & $199.57 \pm 43.15$ & $195.78 \pm 37.44$ & $204.91 \pm 38.18$ & $206.02 \pm 41.16$ & 0.116 & 0.081 \\
\hline MDS & $202.68 \pm 41.42$ & $197.54 \pm 40.90$ & $207.32 \pm 38.23$ & $199.30 \pm 39.34$ & -0.016 & 0.771 \\
\hline HDL choles & $\left(m g d l^{-1}\right)$ & & & & & \\
\hline MDI & $54.83 \pm 13.57$ & $54.63 \pm 11.15$ & $57.60 \pm 12.75$ & $57.11 \pm 15.59$ & 0.023 & 0.240 \\
\hline MDS & $54.49 \pm 12.39$ & $54.56 \pm 11.02$ & $56.96 \pm 13.79$ & $57.93 \pm 15.62$ & 0.118 & 0.033 \\
\hline LDL cholest & $\left(m g d l^{-1}\right)$ & & & & & \\
\hline MDI & $125.49 \pm 40.39$ & $122.54 \pm 33.85$ & $128.26 \pm 34.81$ & $129.37 \pm 38.50$ & 0.107 & 0.126 \\
\hline MDS & $128.22 \pm 38.33$ & $123.07 \pm 37.32$ & $131.83 \pm 36.82$ & $122.78 \pm 34.67$ & -0.035 & 0.534 \\
\hline Triglyceride & $\left.d I^{-1}\right)$ & & & & & \\
\hline $\mathrm{MDI}$ & $96.23 \pm 47.39$ & $93.09 \pm 49.40$ & $95.48 \pm 48.88$ & $97.81 \pm 52.44$ & 0.019 & 0.833 \\
\hline MDS & $99.99 \pm 50.53$ & $99.63 \pm 49.73$ & $92.79 \pm 47.85$ & $92.96 \pm 54.09$ & -0.097 & 0.080 \\
\hline
\end{tabular}

${ }^{1}$ Groups were generated using quartiles from the dietary pattern indexes: MDI and MDS adjusted by confounding covariates.

${ }^{2}$ Bold, significant $P$-values; SD, standard deviation; $n=328$. Q, quartiles; MDI, Mediterranean diet adherence index (expressed in percentage of adherence); MDS, Mediterranean diet score ${ }^{19}$.

also be subject to the influence of these confounders ${ }^{10,14,33,36-41}$. In the present study, because concentrations of triglycerides and other blood lipids were higher in men than in women and it further increased with age, a statistical adjustment was made. However, the possibility of residual confounding despite the adjustments cannot be excluded.

Diet has a quantifiable influence on chronic disease, but evaluating diet is a cumbersome task that depends on, among other factors, the choice of the dietary assessment tool, dedication, consistency and honesty of the study participants, the accuracy of the data collection and entry as well as the completeness of the dietary database used for analysis ${ }^{42}$. The limitation of using self-reported measures of diet in investigating diet-chronic disease relationships is well recognised ${ }^{43}$. This is a main reason for exploring biological indicators as more objective measures of dietary intake ${ }^{9,10}$.

Plasma and/or urinary markers have been widely used to validate intake of specific nutrients ${ }^{9,44}$ or foods ${ }^{45}$ estimated from self-report food measurements. Recently, biomarkers have been recognised as potential tools for describing dietary patterns, since they may provide dietary information that is complementary to self-reported dietary intake because the errors associated with these two tools are not correlated ${ }^{10,11,38,41,46}$.

On one side, evaluating associations between dietary pattern indexes and biomarkers of dietary intake and disease risk in a large sample of subjects could be a key step in their validation $^{40}$. On the other side, dietary patterns could not only modify disease risk by providing exposure to a variety of protective nutrients, but also can contribute to our 
understanding of the mechanisms involved in this interaction $^{9,10,44}$. This makes biomarkers very useful in epidemiological studies, even if a smaller subsample needs to be used to obtain the necessary blood samples ${ }^{44}$.

In the current study, the examined biomarkers were selected not only because they reflect blood levels of nutrients commonly provided by foods characterising the MDP, but also because subclinical deficiencies of these nutrients have been related to the risk for chronic illnesses, such as cardiovascular disease and cancer ${ }^{38,47}$. Examples of such metabolic relationships, extensively described in the literature, include the relationship between high blood levels of folates and the decrease in risk of arteriosclerosis and cardiac ischaemia ${ }^{47}$. However, there seems to be a weaker relationship between serum cholesterol, a risk factor for coronary heart disease and dietary cholesterol, because of the influence of environmental and genetic factors on the serum cholesterol ${ }^{9}$.

Biomarker data are often available in large crosssectional or longitudinal study datasets and despite the sometimes high cost of the biochemical analyses needed, the use of such data might have a smaller overall burden than the collection and analysis of dietary intake data. However, the use of biomarkers also has several drawbacks, such as the fact that their concentrations depend on food intake, as in the case of tocopherol and lipid intake ${ }^{15}$. Measurement errors, such as misclassification and bias 9 , a high cost and the need to use invasive techniques for collection, may provide additional challenges for the use of biomarkers.

Specific challenges with the use of the biomarkers evaluated in this study are outlined below. Blood levels of vitamin $\mathrm{C}$ are good predictors of intake in the normal range, but not of high intakes due to strict kidney regulation ${ }^{40}$. Similarly, blood retinol has a weak relationship with intake because it too has a strict homeostatic control, except in cases of deficiency ${ }^{36,38,48}$. Low-plasma vitamin B12 levels are rare, because the vitamin is abundant in foods of animal origin and is stored in significant amounts in the body ${ }^{49}$. Serum $\alpha$-tocopherol concentrations, although widely used, are only moderately related to vitamin $\mathrm{E}$ intake $\mathrm{e}^{36,38,48}$. Conversely, blood levels of carotenoids and folates show a strong relationship with their intake ${ }^{10}$.

In addition to general problems with biomarkers and dietary pattern indexes ${ }^{6,24,28}$, the present study has several limitations. Firstly, although analyses were controlled for potential biomarker-confounding factors, causality cannot be inferred from the results due to the cross-sectional nature of the study. Secondly, multiple statistical analyses may have resulted in declaring chance associations as significant. Thirdly, although the sample was representative of the studied region and heterogeneous of different social and geographic contexts, it over-represents individuals over age 50 and slightly over-represents women
(58\%). Moreover, even though it is rare to find strong correlations between food data and biomarkers ${ }^{10}$, the small sample size in this study could have limited our ability to find additional or stronger associations.

Another weakness of our study is that not all of the components of the indexes we examined (i.e. fibre) are represented by the selected biomarkers. Although perfect and practical biomarkers do not exist, other biomarkers of a healthful Mediterranean diet profile could be explored, such as homocysteine, insulin, oleic acid, eicosapentaenoic and docosahexaenoic acid, etc. An attempt to identify biomarkers of multidimensional dietary behaviour would be useful.

The new approach of capturing multidimensional food behaviour by the use of dietary patterns for examining associations between biomarkers and diet has several advantages over reliance on analyses of single nutrients or foods ${ }^{29}$. Not only does such an approach allow an easier detection of synergistic effects of nutrients or foods, but also the results can be more easily translated into foodfocused public health recommendations ${ }^{29}$.

More research needs to be conducted regarding the development, selection, modelling and use of appropriate biomarkers. Further work with larger data sets is required before biomarkers can be used as a complementary tool for evaluating the adherence to the MDP. Such data could be useful for building more robust dietary pattern adherence indexes and might further provide clues for potential physiological mechanisms that explain the beneficial effects on chronic disease risk associated with the MDP.

\section{Acknowledgements}

This study was supported by the Mediterranean Diet Foundation. Data provided by courtesy of Department of Health, Government of Catalonia. We thank Konstantina Skaltsa from the Public Health Department of the University of Barcelona for her help and support in the statistical analysis. None of the authors had any conflicts of interest.

\section{References}

1 Serra-Majem L, Roman B, Estruch R. Scientific evidence of interventions using the Mediterranean diet: a systematic review. Nutrition Reviews 2006; 64: 531-52.

2 Keys A, Menotti A, Karvonen M. The diet and 15-year death rate in the Seven Countries study. American Journal of Epidemiology 1986; 124: 903-15.

3 Kant AK. Dietary patterns and health outcomes. Journal of American Dietetic Association 2004; 104: 615-35.

4 Hu F. The Mediterranean diet and mortality-olive oil and beyond. New England Journal of Medicine 2003; 348: 2595-6.

5 Hu F. Dietary pattern analysis: a new direction in nutritional epidemiology. Current Opinion in Lipidology 2002; 13: $3-9$. 
6 Bach-Faig A, Serra-Majem L, Carrasco JL, Roman B, Ngo J, Bertomeu I, Obrador B. The use of indexes evaluating the adherence to the Mediterranean diet in epidemiological studies: a review. Public Health Nutrition 2006; 9: $132-46$.

7 Serra-Majem L, Ngo de la Cruz J, Trichopoulou A. Dieta Mediterránea. In: Serra Majem L, Aranceta Bartrina J, eds. Nutrición y Salud Pública. Métodos, Bases científicas y Aplicaciones, 2nd ed. Barcelona: Editorial Masson, 2006; 642-56.

8 Kant A. Indexes of overall diet quality: a review. Journal of American Dietetic Association 1996; 96: 785-91.

9 Hunter D. Biochemical indicators of dietary intake. In: Willett W, ed. Nutritional Epidemiology. New York: Oxford University Press, 1990; 143-216.

10 García-Closas R. Indicadores bioquímicos de la ingesta dietética. In: Serra-Majem L, Aranceta J, Mataix J, eds. Nutrición y Salud pública. Método, Bases científicas y Aplicaciones. Barcelona: Editorial Masson, 1995; 141-54.

11 Zamora-Ros R, Urpi-Sarda M, Lamuela-Raventos RM, Estruch R, Vázquez-Agell M, Serrano-Martínez M, Jaeger W, AndresLacueva C. Diagnostic performance of urinary resveratrol metabolites as biomarker of moderate wine consupmtion. Clinical Chemistry 2006; 52(7): 1373-80.

12 Serra-Majem L, Ribas L, García-Closas R, Ramon J, SalvadorCastell G, Farran-Codina A, et al. Avaluació de l'estat nutricional de la població catalana (1992-1993). Barcelona: Departament de Sanitat i Seguretat Social, 1996.

13 García-Closas R, Serra-Majem L, Chacón-Castro P, OlmosCastellvell M, Ribas-Barba L, Salleras-Sanmartí L, et al. Serum lipid levels in a representative sample of adult individuals from Catalonia, Spain. Medicina Clinica (Barcelona) 1999; 113: 6-12.

14 García-Closas R, Serra-Majem L, Sabater-Sales G, OlmosCastellvell M, Ribas-Barba L, Salleras-Sanmartí L, et al. Distribution of the serum concentration of vitmain C, folic acid and vitamin B12 in a representative sample of the adult population of Catalonia (Spain). Medicina Clinica (Barcelona) 2002; 118(4): 135-41.

15 García-Closas R, Serra-Majem L, Cruz-Pastor F, OlmosCastellvell M, Roman B, Ribas-Barba L, Salleras-Sanmartí L, et al. Distribution of the serum concentration of $\beta$-carotene, retinol and $\alpha$-tocopherol in a representatives ample of the adult population of Catalonia (Spain). Medicina Clinica (Barcelona) 2002; 118(7): 256-61.

16 Fidanza F. Who remembers the true Italian Mediterranean diet? Diabetes, Nutrition \& Metabolism 2001; 14(3): 119-20.

17 Farran A, Zamora R, Cervera P \& (CESNID). Tablas de Composición de alimentos del CESNID. Barcelona: McGrawHill/Interamericana de España, S.A.U./Edicions Universitat de Barcelona, 2004

18 Sánchez-Villegas A, Martínez J, de Irala J, Martínez-González M. Determinants of the adherence to an 'a priori' defined Mediterranean dietary pattern. European Journal of Nutrition 2002; 41: 249-57.

19 Trichopoulou A, Costacou T, Bamia C, Trichopoulos D. Adherence to a Mediterranean diet and survival in a Greek population. New England Journal of Medicine 2003; 348: 2599-608.

20 Tur J, Romaguera D, Pons A. Adherence to the Mediterranean dietary pattern among the population of the Balearic Islands. British Journal of Nutrition 2004; 92: 341-6.

21 Stampfer MJ, Colditz GA, Willett WC, Speizer FE, Hennekens $\mathrm{CH}$. A prospective study of moderate alcohol consumption and the risk of coronary disease and stroke in women. The New England Journal of Medicine 1988; 319(5): 267-73.

22 Rimm EB, Giovannucci EL, Willett WC, Colditz GA, Ascherio A, Rosner B, Stampfer MJ. Prospective study of alcohol consumption and risk of coronary disease in men. Lancet 1991; 338(8765): 464-8.
23 WHO. Guidelines for Controlling and Monitoring the Tobacco Epidemic. Geneva: Tobacco or Health Programme, 1997.

24 Kant AK, Graubard BI. A comparison of Three Dietary Pattern Indexes for predicting biomarkers of diet and disease. Journal of the American College of Nutrition 2005; 24(4): 294-303.

25 Gerber M. Qualitative methods to evaluate Mediterranean diet in adults. Public Health Nutrition 2006; 9(1A): 147-51.

26 Neuhouser ML, Patterson RE, King IB, Horner NK, Lampe JW. Selected nutritional biomarkers predict diet quality. Public Health Nutrition 2003; 6(7): 703-9.

27 Schulze MB, Hoffmann K. Methodological approaches to study dietary patterns in relation to risk of coronary heart disease and stroke. British Journal of Nutrition 2006; 95 : $860-9$.

28 Fung TT, McCullough ML, Newby PK, Manson JE, Meigs JB, Rifai N, Willett WC, Hu FB. Diet-quality scores and plasma concentrations of markers of inflammation and endothelial dysfunction. The American Journal of Clinical Nutrition 2005; 82: 163-73.

29 Fung TT, Rimm EB, Spiegelman D, Rifai N, Tofler GH, Willett WC, Hu FB. Association between dietary patterns and plasma biomarkers of obesity and cardiovascular disease. The American Journal of Clinical Nutrition 2001; 73: 61-7.

30 Hann CC, Rock CL, King I, Drewnowski A. Validation of the Healthy Eating Index with use of plasma biomarkers in a clinical sample of women. The American Journal of Clinical Nutrition 2001; 74: 479-96.

31 Newby PK, Hu FB, Rimm EB, Smith-Warner SA, Feskanich D, Sampson L, Willett WC. Reproducibility and validity of the Diet Quality Index Revised as assessed by use of a foodfrequency questionnaire. The American Journal of Clinical Nutrition 2003; 78(5): 941-9.

32 Gerber MJ, Scali JD, Michaud A, Durand MD, Astre CM, Dallongeville J, Romon MM. Profiles of a healthful diet an dits relatinoship to biomarkers in a population sample form Mediterranean southern France. Journal of the American Dietetic Association 2000; 100: 1164-71.

33 Rock CL, Thornquist MD, Kristal AR, Patterson RE, Cooper D, Neuhouser MI, et al. Demographic and lifestyle variables differentially explain variability in serum caroteinoids and fat-soluble vitamins: baseline results from the Olestra Post. Marketing Surveillance Study. Journal of Nutrition 1999; 129: 855-64.

34 González C, Argílaga S, Agudo A, Amiano P, Barricarte A, Beguiristain JM, et al. Sociodemographic differences in adherence to the Mediterranean dietary pattern in Spanish populations. Gaceta Sanitaria 2002; 16: 214-21.

35 Schröder H, Marrugat J, Vila J, Covas M, Elosua R. Adherence to the traditional Mediterranean diet is inversely associated with body mass index and obesity in Spanish population. Journal of Nutrition 2004; 134: 3355-61.

36 Gibson RS. Nutritional Status Assessment. A Manual for Population Studies. London: Chapman and Hall, 1991.

37 Faruque MO, Khan MR, Rahman MM, Ahmed F. Relationship between smoking and antioxidant nutrient status. British Journal of Nutrition 1995; 73(4): 625-32.

38 Willett W. Nutritional Epidemiology, 2nd ed. New York: Oxford University Press, 1998.

39 Cafolla A, Dragoni F, Girelli G, Tosti ME, Costante A, Pastorelli D, et al. Folate status in Italian blood donors: relation to gender and smoking. Haematologica 2000; 85: 694-8.

40 Fidanza F. Nutritional Status Assessment. A Laboratory Manual. New York: Oxford University Press, 1993.

41 Prentice RL, Sugar E, Wang CY, Neuhouser M, Patterson R. Research strategies and the use of nutrient biomarkers in studies of diet and chronic disease. Public Health Nutrition 2002; 5(6A): 977-84. 
42 Thompson FE, Byers T. Dietary assessmet resource manual. Journal of Nutrition 1994; 124(11): 2245-317.

43 Kipnis V, Midthune D, Freedman LS, Bingham S, Schatzikin A, Subar A, et al. Empirical evidence of correlated bias in dietary assessment instruments and its implications. American Journal of Epidemiology 2001; 153: 394-403.

44 Bingham SA. Biomarkers in nutritional epidemiology. Public Health Nutrition 2002; 5(6A): 821-7.

45 Drewnowski A, Rock CL, Henderson SA, Shore AB, Fischler C, Galan P, et al. Serum beta-carontene and vitamin C as biomarkers of vegetable and fruit intakes in a community- based sample of French adults. American Journal of Clinical Nutrition 1997; 65: 1796-802.

46 Margetts BM, Nelson M, ed. Design Concepts in Nutritional Epidemiology. Oxford: Oxford University Press, 1997.

47 WCRF. Nutrition and the Prevention of Cancer: A Global Perspective. Washington, DC: World Cancer Research Fund and American Institute for Cancer Prevention, 1997.

48 Fidanza F. Nutritional Status Assessment. A Manual for Population Studies. Londres: Chapman and Hall, 1991.

49 Gibson RS. Nutritional Assessment. A Laboratory Manual. New York: Oxford University Press, 1993. 